\title{
Traumatic Fibroma: A Case Series
}

\section{${ }^{1}$ Navnita Singh, ${ }^{2}$ Shivaprasad Bilichodmath, ${ }^{3}$ Savita Sambhashivaiah}

\begin{abstract}
Aims: This article addresses the clinical presentation, diagnosis, histological features, and treatment of two cases of fibroma.

Background: Fibromas are proliferative fibrous lesions of the gingiva and oral mucosa that may cause esthetic and functional problems. Fibrous hyperplasia and fibroepithelial hyperplasia are histological variants of these nonneoplastic lesions.
\end{abstract}

Case description: Case 1 demonstrated an overgrowth in the lower back tooth region. Case 2 was present at the corner of the lip mucosa. Both the growths were pale in color, sessile, painless, and firm in consistency. Lesions caused discomfort during chewing of food. Thus, both the lesions were excised using diode laser in continuous mode and sent for histopathological assessment.

Conclusion: These lesions are a result of trauma/chronic irritation and arise from cells of oral mucous membrane or periosteum.

Clinical significance: The cases demonstrate the need for proper diagnosis, role of biopsy, and histologic evaluation in the management of these lesions.

Keywords: Case series, Diode laser, Fibroepithelial hyperplasia, Traumatic fibroma.

How to cite this article: Singh N, Bilichodmath $S$, Sambhashivaiah S. Traumatic Fibroma: A Case Series. J Health Sci Res 2016;7(1):28-31.

\section{Source of support: Nil}

Conflict of interest: None

\section{INTRODUCTION}

Fibroma is considered the most common benign growth in the oral cavity. ${ }^{1}$ They may arise from the gingival connective tissue or from the periodontal ligament. They are slow-growing, oval tumors that are firm and nodular, but few cases have been reported to be soft and vascular. Found in $1.2 \%$ of adults, this inflammatory hyperplasia is the most common tissue biopsy arising from the oral cavity and usually composed of types I and III collagen. Gingival lesions are also common, although at gingiva they are a result of chronic infection rather than

\footnotetext{
${ }^{1}$ Postgraduate Student, ${ }^{2}$ Reader, ${ }^{3}$ Principal, Professor and Head

${ }^{1-3}$ Department of Periodontology, RajaRajeswari Dental College and Hospital, Bengaluru, Karnataka, India
}

Corresponding Author: Navnita Singh, Postgraduate Student, Department of Periodontology, RajaRajeswari Dental College and Hospital, Bengaluru, Karnataka, India, Phone: +918197600464, e-mail: navnita17@gmail.com trauma. ${ }^{2}$ The most common clinical aspect is the growth of a well-delimited smooth-surfaced tissue, usually of a normal-colored mucosa, sessile or pedunculated base, of hard consistency, ${ }^{3}$ and smaller than $1.5 \mathrm{~cm}$ at its largest diameter, ${ }^{4}$ though there have also been reports of injury larger than $1.5 \mathrm{~cm} .{ }^{5}$ The term "focal fibrous hyperplasia," as suggested by Daley et al 1990, ${ }^{6}$ implies "a reactive tissue response, is preferable to the term, fibroma" which implies incorrectly, a benign neoplastic proliferative fibrous connective tissue. ${ }^{7}$ The lesion presents as painless, sessile, round or ovoid broad-based swelling, lighter in color than the surrounding tissue due to a reduced vascularity. ${ }^{8}$ Treatment of the fibroma involves surgical excision using scalpel, electrocautery, laser, and recurrences are very infrequent. This article explains about the diagnosis and treatment planning for two cases of traumatic fibroma.

\section{CASE REPORTS}

\section{Case 1}

A 32-year-old male patient reported to Department of Periodontology, RajaRajeswari Dental College and Hospital, with the chief complaint of swelling in the right lower back tooth region since 2 months. History revealed a systemically healthy male with no family history of gingival epulis. The lesion was first noticed 2 months ago and the size gradually increased. There was no history of dental and/or facial trauma. The lesion was painless on digital pressure. There was no history of bleeding, except on digital pressure and use of interdental aids. Oral hygiene status was found to be good with an OHI-S score of 1 . Patient visited a private clinic 1.5 months ago and underwent a supragingival scaling.

\section{Examination of Gingiva}

The lesion on the buccal gingiva of about $10 \times 7 \mathrm{~mm}$ in size, sessile, pink in color, in the region of 48 (Fig. 1). The lesion was mildly erythematous and firm in consistency. Radiograph of the area showed no bony involvement. Phase I therapy with supragingival scaling was performed. The patient was prescribed chlorhexidine mouthwash twice daily for 1 week. Then, excisional biopsy was performed using diode laser at $3 \mathrm{~W}$ in a continuous mode (Figs 2 and 3). Figure 2 shows immediate postoperative photograph. Ibuprofen $400 \mathrm{mg}$ three times a day for 3 days was prescribed to the patient postoperatively. Postoperative healing was found to be uneventful. Figure 4 


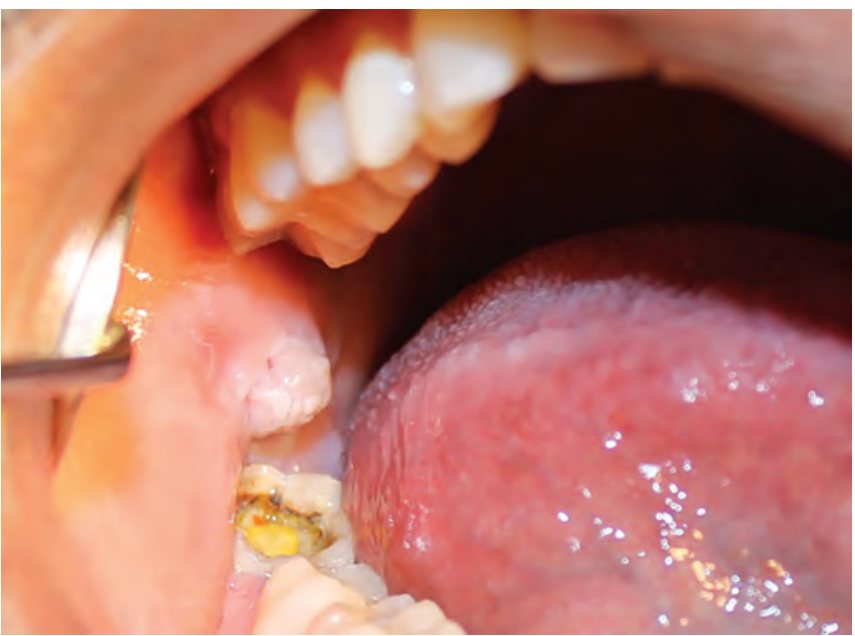

Fig. 1: Preoperative photograph

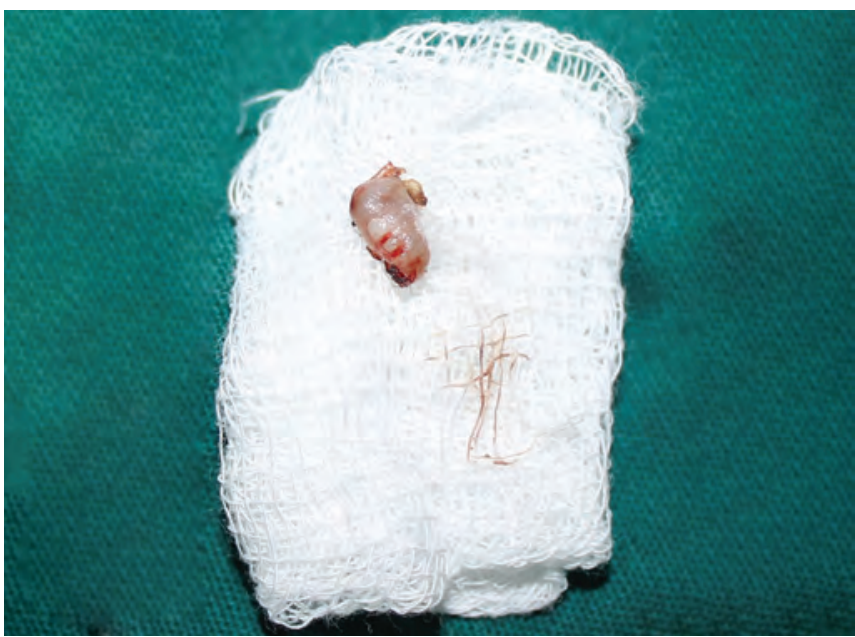

Fig. 3: Excised tissue of case 1

shows 1 month after healing. No pain or discomfort or difficulty in eating was reported.

\section{Differential Diagnosis}

Hyperplastic fibroma, peripheral giant cell granuloma, peripheral ossifying fibroma, pyogenic granuloma, papilloma, gingival cyst were considered in differential diagnosis.

\section{Histopathology}

H\&E stained section showed epithelium and connective tissue. Epithelium was orthokeratinized stratified squamous epithelium of varying degree. Underlying fibrous connective tissue stroma showed mild chronic inflammatory infiltrate and few blood vessels. These features are suggestive of fibroma (Fig. 5).

\section{Case 2}

A 55-year-old male patient reported to Department of Periodontology, RajaRajeswari Dental College and Hospital, with the chief complaint of swelling in the

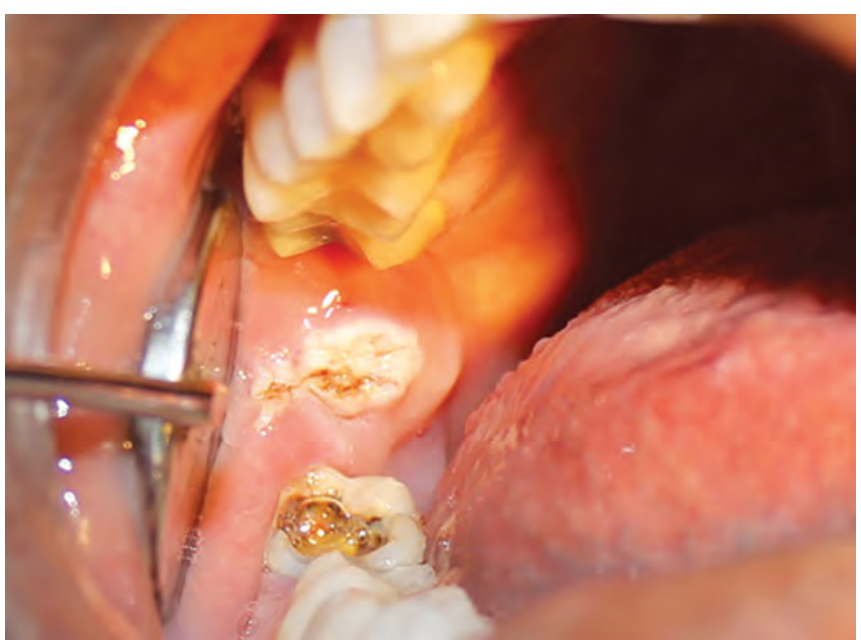

Fig. 2: Immediate postoperative after laser excision

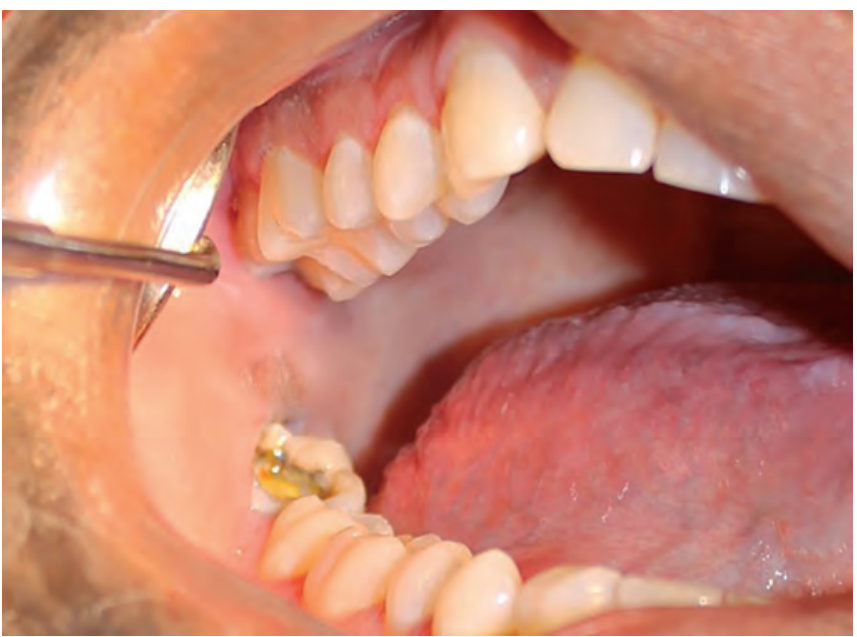

Fig. 4: One month postoperative of case 1

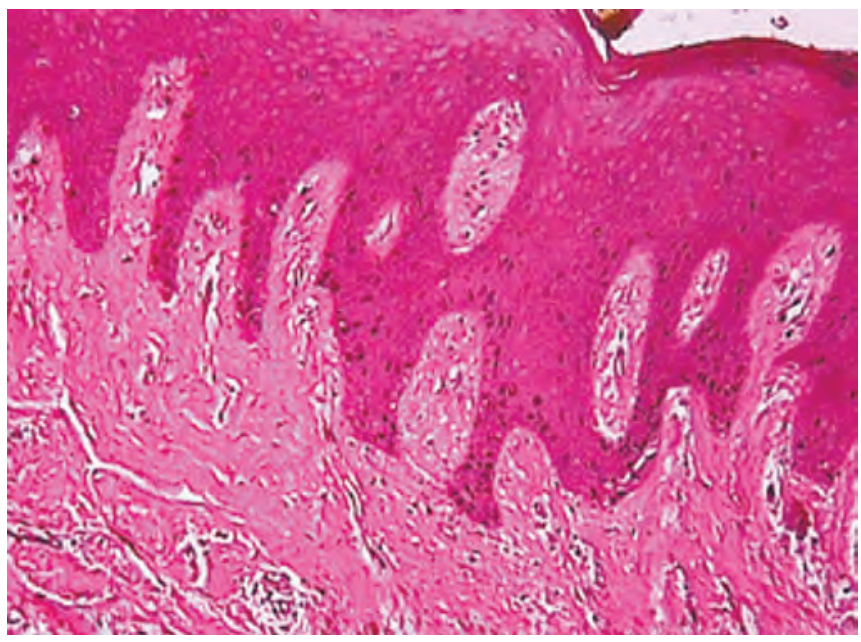

Fig. 5: Histolopathological section showing orthokeratinized hyperplastic epithellium of case 1

right corner of the lip region. The lesion was first noticed 4 months ago, which increased in size gradually. Any history of dental and/or facial trauma was not reported. The lesion was found to be painless except on digital pressure. His oral hygiene was acceptable with an OHI-S 


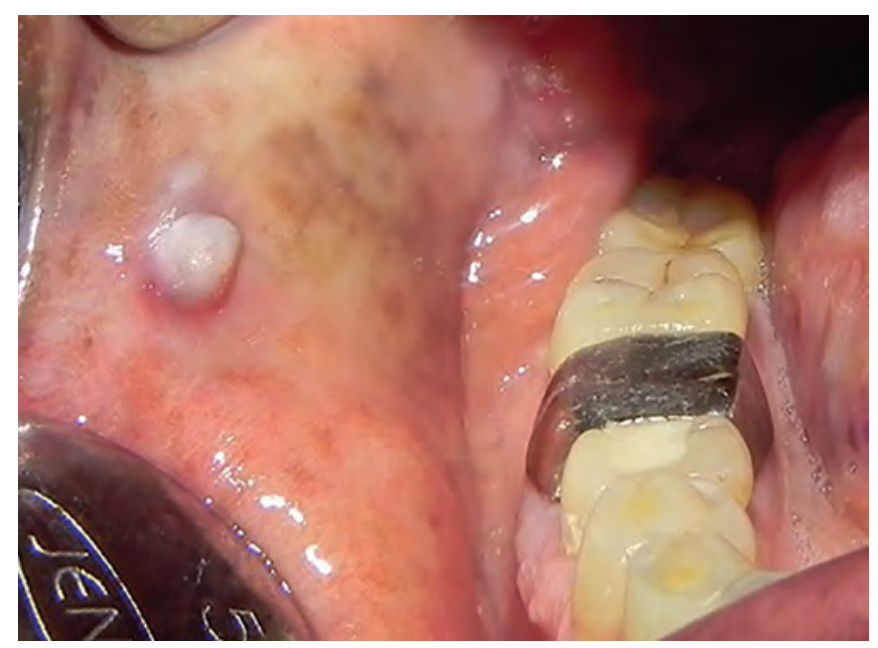

Fig. 6: Preoperative photo of case 2

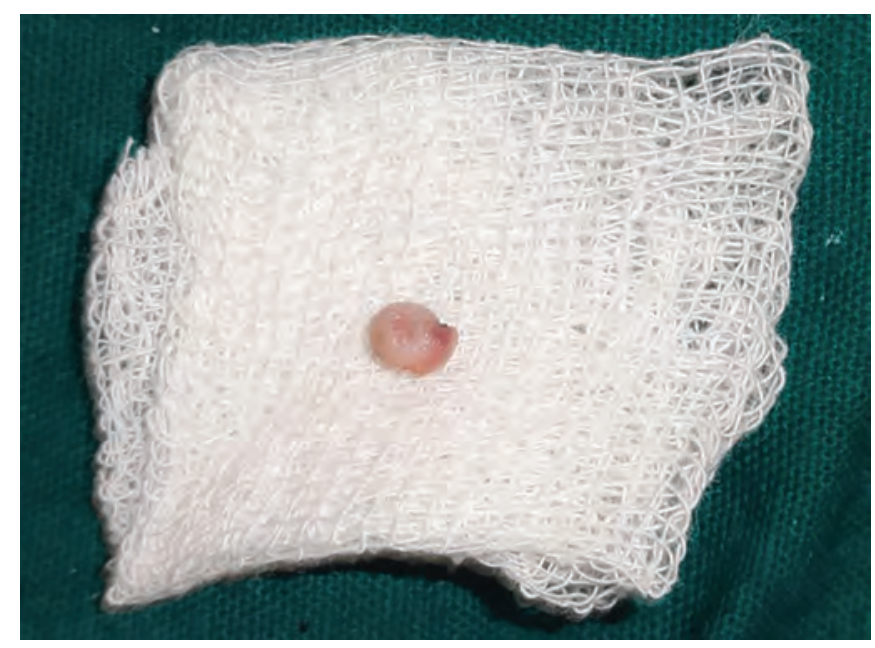

Fig. 8: Excised tissue of case 2

score of 1.4. He had not undergone any dental procedures in the past 1 year.

\section{Examination of the Soft Tissues}

The lesion was about $4 \times 5 \mathrm{~cm}$ in size, pink in color, at the corner of the mouth in the region of 13 and 43 (Fig. 6). The lesion was firm in consistency and sessile. Radiograph of the area revealed no bony involvement. Phase I therapy with supragingival scaling was carried out and chlorhexidine mouthwash was prescribed twice daily for 1 week. Then, excision biopsy was performed using diode laser at $3 \mathrm{~W}$ power in continuous mode (Fig. 7). Figure 8 shows the fibrous tissue following excision. One-month follow-up shows complete healing with no complication (Fig. 9). It was not associated with any pain or discomfort or difficulty in eating.

\section{Differential Diagnosis}

Hyperplastic fibroma, mucocele, peripheral giant cell granuloma, peripheral ossifying fibroma, pyogenic granuloma, papilloma, were considered in differential diagnosis.

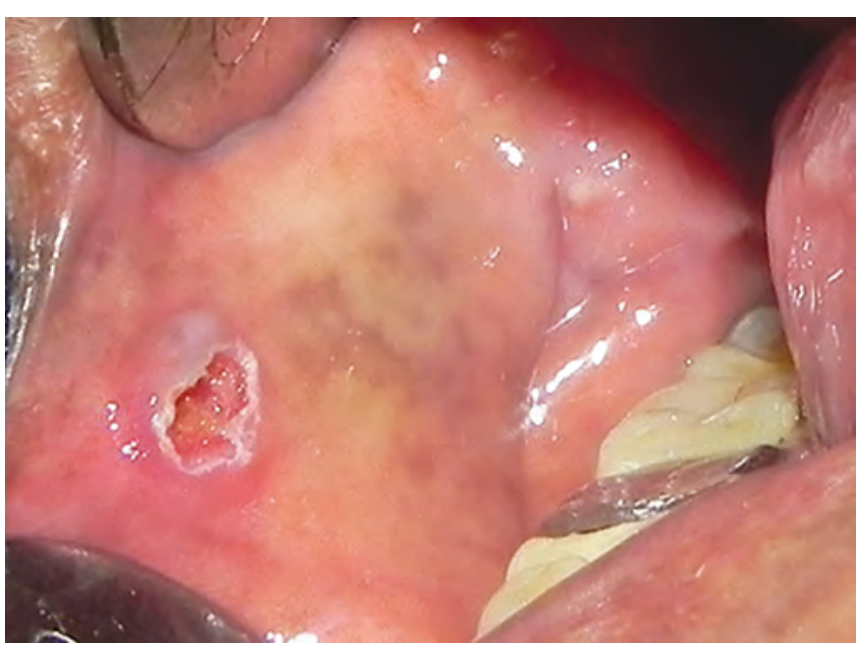

Fig. 7: Immediate postoperative photo of case 2

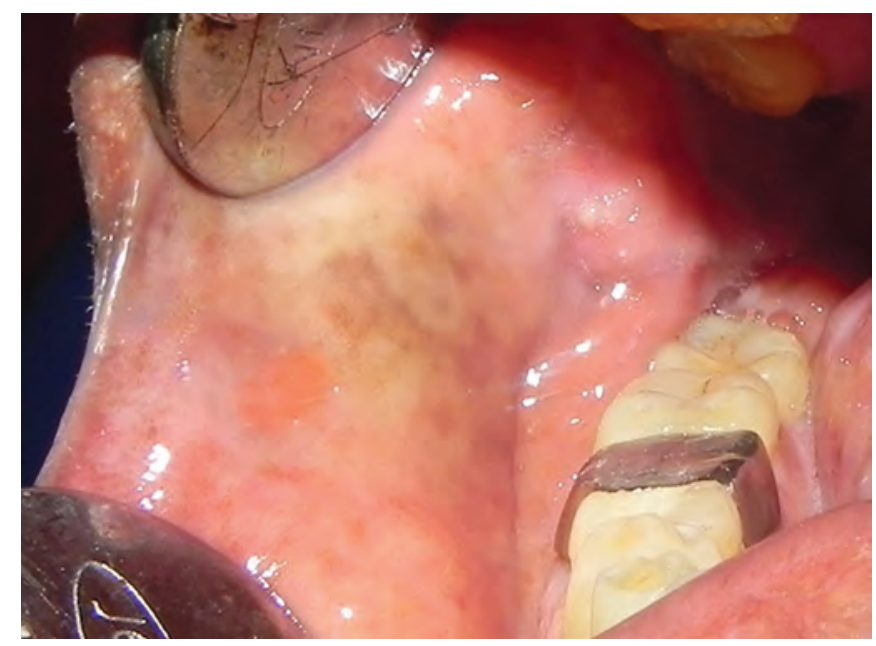

Fig. 9: One month postoperative of case 2

\section{Histopathology}

H\&E stained sections showed parakeratinized stratified squamous type of epithelium and connective tissue. Epithelium was found to be hyperplastic. Connective tissue contained numerous blood vessels with some surrounded by plump and proliferating endothelial cells. Dense chronic inflammatory infiltrate comprising of lymphocytes, plasma cells, and macrophages is present (Fig. 10).

\section{DISCUSSION}

A diverse group of pathologic processes can produce tissue enlargement of the oral cavity. Thus, those lesions often present as a diagnostic challenge. Within these lesions, a group of reactive hyperplasia that develop in response to a chronic, recurring tissue injury stimulates an exuberant or excessive tissue repair response. ${ }^{9}$ Reactive gingival lesions have been classified into pyogenic granuloma, peripheral giant cell granuloma, fibrous hyperplasia, and peripheral fibroma with calcification by Kfir et al..$^{10}$ As the most common nonneoplastic growth in the 


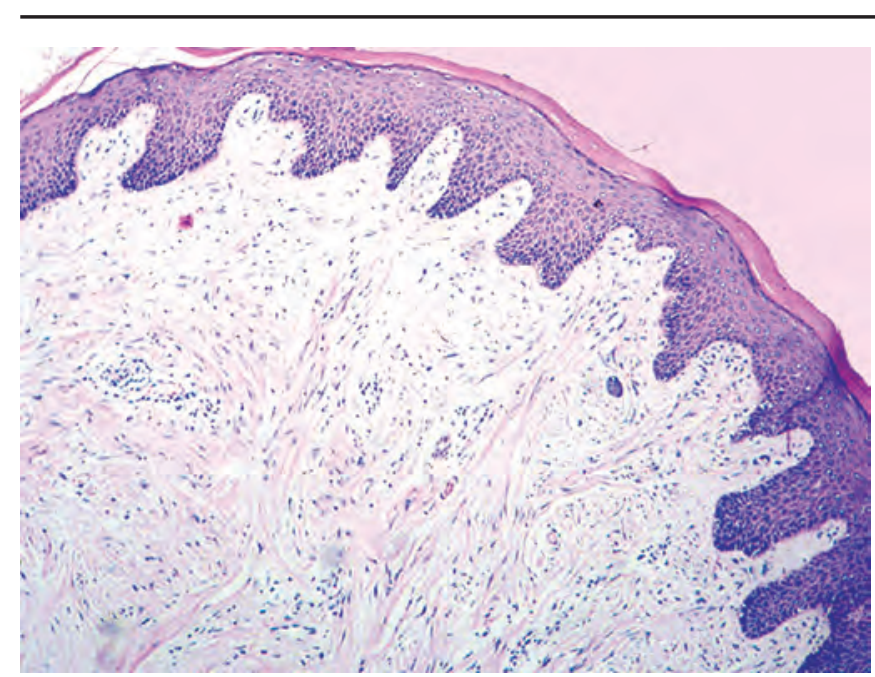

Fig. 10: Histopatological section showing parakeratinized epithelium with hyperplastic epithellium and connective tissue in case 2

oral cavity, much has been written about the fibroma. It has been known as irritation fibroma, traumatic fibroma, fibrous hyperplasia, focal fibrous hyperplasia, localized hyperplasia, fibrous polyp ${ }^{11}$ and fibroepithelial polyp. The clinical presentation and epidemiology of most nonneoplastic growths in the oral cavity are quite similar; thus, identification is dependent on histopathological differentiation. Histologically, these lesions vary from granulation tissue to mature scar-like tissue, depending on age and vascularity. Lesions are collagenous and composed of mature fibrous tissue with prominent vascular pattern. Epithelial changes also correlate with the lesion's age and degree of inflammation. Fibroepithelial hyperplasias, when inflamed, are covered by uniformly hyperplastic epithelium, with arcading rete pattern when ulcerated. The formulation of a differential diagnosis requires identification of any reactive hyperplastic gingival lesion to enable accurate patient evaluation and management. It is important to differentiate these lesions clinically and histologically from precancerous, developmental, and neoplastic lesions. Differential diagnoses include gingival non-Hodgkin's lymphoma angiosarcomas, metastatic tumors in the oral cavity, Kaposi's sarcoma, and hemangioma. ${ }^{12}$ In the present case series, patients did not reveal any specific history of trauma to oral mucosa. Clinical findings suggest that occlusal forces might be the cause of traumatic fibroma in these cases.

\section{CONCLUSION}

For the myriad histological patterns observed in reactive hyperplasia, connective tissue response to varied intensities of gingival irritation may be responsible. So, it is of prime importance to distinguish between hyperplasia and neoplasia as neoplasias are not selflimiting conditions and long-standing hyperplastic lesions in presence of chronic irritation can get converted to neoplasia. In addition to the physical characteristics of the lesion, the patient's demographics, presence of associated symptoms, related systemic disorders, and location and growth patterns of the lesion all give clues to adequately diagnose and treat their typical histopathologic architecture.

\section{REFERENCES}

1. Esmeili T, Lozada-Nur F, and Epstein J. "Common benign oral soft tissue masses," Dental Clinics of North America, vol. 49, no. 1, pp. 223-240, 2005.

2. Carranza's Clinical Periodontology 11 ed.

3. Baumgartner JC, Stanley HR, Salomone JL. Peripheral ossifying fibroma. J Endodont 1991;17:182-185.

4. Kfir Y, Buchner A, Hansen LS. Reactive lesions of the gingival - A clinicopathologic study of 741 cases. J Periodontol 1980;51:655-661.

5. Kendrick F, Waggoner WF. Managing peripheral ossifying fibroma. J Dent Child 1996, 63:35-138.

6. Daley TD, Wysocki GP, Wysocki PD, Wysocki DM. The major epulides: clinicopathological correlations. J Can Dent Assoc 1990;56:627-630.

7. Dieter K, Andreas A, Christos C. Reactive hyperplasias, precancerous and malignant lesions of the oral mucosa. JDDG2008;6:217-232.

8. Ramzi C, Vinay K, Tucker C. Robbins pathologic basis of disease. 6th ed. W.B. Saunders. 1999.

9. Mohammed NA, Chandrasekaran SC, Mohan V. Fibroma of the Gingiva: a case report of a 20 year old lesion. Int J Contemp Dent 2010;1:107-109.

10. Kfir Y, Buchner A, Hansen LS. Reactive lesions of the gingiva. A clinic-pathological study of 741 cases. J Periodontol 1980; 51:655-661.

11. Gustafson BA, Greenspan JS. Multiple polypoid conditions of the oralmucosa. British J Oral Surg 1974;12:91-95.

12. Riya V, Amitha R, Rahul B, Biju T, Nishita L. Philip. Fibroma - A misnomer: case Series. NUJHS 2015;5(4):83-87. 